\title{
Association of measures of fetal and childhood growth with non-clinical psychotic symptoms in 12-year-olds: the ALSPAC cohort
}

K. Thomas, G. Harrison, S. Zammit, G. Lewis, J. Horwood, J. Heron, C. Hollis, D. Wolke, A. Thompson and D. Gunnell

\section{Background}

Previous studies have suggested that impaired fetal and childhood growth are associated with an increased risk of schizophrenia, but the association of pre-adult growth with non-clinical psychotic symptoms (psychosis-like symptoms) in children is not known.

\section{Aims}

To explore the associations of body size at birth and age 7.5 years with childhood psychosis-like symptoms.

\section{Method}

Prospective cohort of children followed up from birth to age 12: the ALSPAC cohort.

\section{Results}

Data on 6000 singleton infants born after 37 weeks of gestation. A one standard deviation increase in birth weight was associated with an $18 \%$ reduction in the risk of definite psychosis-like symptoms after adjusting for age and gestation (Odds ratio $(\mathrm{OR})=0.82,95 \% \mathrm{Cl}=0.73-0.92, P=0.001$ ). This association was partly confounded by maternal anthropometry, smoking during pregnancy, socioeconomic status and IQ. A similar association was seen for birth length and psychosis-like symptoms, which disappeared after controlling for birth weight. There was little evidence for an association of 7-year height or adiposity with psychosis-like symptoms.

\section{Conclusions}

Measures of impaired fetal, but not childhood, growth are associated with an increased risk of psychosis-like symptoms in 12-year-olds.

\section{Declaration of interest}

None.
There is growing evidence that non-clinical psychotic symptoms such as auditory and visual hallucinations are commonly experienced in childhood ${ }^{1}$ and adulthood. ${ }^{2,3}$ These findings suggest that psychosis may be a continuous phenotype only rarely presenting as a severe clinical disorder. However, the presence of non-clinical psychotic symptoms (psychosis-like symptoms) in childhood does appear to signal a greatly increased risk of adult psychosis. In the Dunedin cohort, $14 \%$ of 11 -year-olds reported experiencing at least one weak or strong psychotic symptom; children with weak symptoms were five times more likely than their peers to develop schizophreniform disorder at age 26, and children with strong symptoms were 16 times more likely. ${ }^{1}$ Similarly, among 18- to 64-year-olds in the Dutch NEMESIS study, individuals reporting incident (non-clinical) psychotic symptoms were at 65 -fold greater risk of developing clinical psychosis over a 2 -year follow-up. ${ }^{4}$ We have recently measured psychosis-like symptoms in the Avon Longitudinal Study of Parents and Children (ALSPAC); 13.7\% of 12-year-olds were rated as having experienced at least one psychotic symptom in the previous 6 months. ${ }^{5}$

More research is needed to confirm both the aetiology and long-term health consequences of psychosis-like symptoms in childhood. Analysis of the Dunedin cohort found that the risk of psychosis-like symptoms at age 11 was higher among children with low IQ and delayed motor or language development, suggesting that psychosis-like symptoms may be part of a pan-developmental spectrum of impairments associated with schizophreniform disorder. ${ }^{6}$

A number of studies have found associations between measures of restricted fetal and childhood growth and later risk of psychosis. ${ }^{7-9}$ Such associations are thought to indicate that factors affecting pre-adult growth may also influence neurodevelopment and, in turn, the risk of psychosis. The investigation of such factors in relation to psychosis-like symptoms will both provide evidence of relevance to the neurodevelopmental model of psychosis ${ }^{10}$ and further clarify the relevance of psychosis-like symptoms to the study of the aetiology of psychosis. Here, we investigate the association of birth weight, birth length, childhood height and body mass index (BMI) with psychosis-like symptoms at age 12 and whether any association between these measures and psychosis-like symptoms is mediated through their association with IQ. Our research was conducted on members of a large population-based birth cohort, ALSPAC. The cohort was originally established in the early 1990s to investigate how genotype combines with the environment to influence health and development.

\section{Method}

\section{Participants}

The ALSPAC study (www.alspac.bris.ac.uk) is a longitudinal cohort study following children who had an expected date of delivery between April 1991 and December 1992, and were resident in the former Avon Health Authority area (centred on Bristol, UK) at the time of their birth. The study has been described in detail previously; ${ }^{11}$ its aim is to study multiple outcomes including changes in anthropometry, attitudes and behaviour, fitness and other cardiovascular risk factors and mental health. The participants' parents have completed regular postal questionnaires about all aspects of their child's health and development. Since the children were 7.5 years of age, they have been invited to attend annual assessment clinics where they participate in a range of face-to-face interviews and physical tests. The initial sample consisted of 14541 pregnancies, which resulted in 14062 live births. The current study examines data 
obtained from 6455 children who attended an assessment clinic, at mean age 12.8 years (s.d. $=0.2$ ).

\section{Ethical approval}

Ethical approval for the study was obtained from the ALSPAC Law and Ethics Committee and the local research ethics committees.

\section{Non-clinical psychotic symptoms}

Non-clinical psychotic symptoms (psychosis-like symptoms) were measured at the ALSPAC 12-year clinic using the PLIKSi semistructured face-to-face interview. ${ }^{5}$ Psychology graduates rated the children on whether they had experienced any of 12 main items of psychotic symptoms: hallucinations (visual and auditory); delusions (delusions of being spied on, persecution, thoughts being read, reference, control, grandiose ability and other unspecified delusions); and bizarre delusions (thought broadcasting, insertion and withdrawal). The items were assessed to be: not present, suspected or definitely present. The agreement between interviewers was good; the average kappa value $(\kappa)$ for interrater reliability was 0.72 .

Two main psychosis-like symptom outcomes are considered in this paper: whether any of the 12 items were assessed as being suspected or definitely present at the 12-year interviews; and a narrower outcome of definite symptoms only. As secondary analyses we also examined associations specifically with third person auditory hallucinations and 'first rank' delusions (delusions of control or delusions of thought broadcast, insertion or control). These two groups of symptoms were also combined as narrowly defined psychosis-like symptoms; these symptoms are considered to be more characteristic of schizophrenia, in accordance with both DSM-IV ${ }^{12}$ and ICD-10 ${ }^{13}$ criteria. Finally, we examined frequently occurring definite psychosis-like symptoms without attributions i.e. any definite symptoms occurring monthly or more often, which did not occur during a state of going to sleep, waking from sleep, fever or directly following any substance misuse.

\section{Measures of growth and adiposity}

Birth weights were extracted from hospital records and birth length (crown to heel) was measured by ALSPAC staff using a Harpenden neonatometer (Holtain Ltd, www.anthropometer. com). The ponderal index, a measure of infant adiposity, was calculated as $100 \times$ birth weight $(\mathrm{g}) /$ birth length $\left(\mathrm{cm}^{3}\right)$.

All participants were invited to attend a health examination at age 7.5 years. Standing height was measured to the nearest $0.1 \mathrm{~cm}$, using a Harpenden stadiometer as described by Cameron. ${ }^{14}$ Weight was measured to the nearest $50 \mathrm{~g}$ using Tanita weighing scales (Tanita UK Ltd, Uxbridge). Body mass index was calculated from the height and weight measurements (weight $(\mathrm{kg}) /$ height $\left(\mathrm{m}^{2}\right)$ ). Waist circumference was measured to the nearest $\mathrm{mm}$ at the minimum circumference of the abdomen between the iliac crests and the lowest rib, using the Harpenden anthropometric tape.

All weight and length measurements were considered in quintiles calculated from the data or recognised categories and gender-specific $z$-scores, as appropriate. For the measures at 7.5 years, the $z$-scores were estimated from models that also took account of the age (in months) of the children when they were measured.

\section{Potential confounders}

Gestation was estimated from the date of last menstrual period and was included in the models as a continuous variable. Resuscitation at birth (yes or no) - a measure of likely asphyxia
- was obtained from birth records. Questionnaires during the antenatal period provided information on further potential confounders: maternal parity (zero, one, two or more); mother's pre-pregnancy height and weight (considered as continuous measures); paternal height and weight (considered as continuous measures); maternal and paternal age (in years); maternal smoking during pregnancy (number of cigarettes smoked per day); household crowding (number of people in the household/ number of rooms in the home); family history of schizophrenia (parental report that either they or the grandparents of the study child had schizophrenia); parental social class was assessed based on the lower of the mother or partner's occupational social class (using the 1991 OPCS classification ${ }^{15}$ and dichotomising into manual and non-manual); housing tenure (mortgaged/owned, council rented or rented/other); and maternal education (less than O-level, O-level, or more than O-level, where O-levels were the standard school-leaving qualifications taken around age 16 years until recently in the UK).

An abbreviated form of the Wechsler Intelligence Scale for Children (WISC)-III (UK version) was used to measure the IQ of the children at the ALSPAC 8-year clinic. ${ }^{16}$ We included this as a continuous variable in multivariable models to investigate whether any association between measures of growth and psychosis might be mediated through the association of fetal growth with IQ, ${ }^{17}$ a known risk factor for psychosis. ${ }^{18,19}$

\section{Statistical analyses}

All analyses were carried out using SAS version 8 for Windows. Analysis was restricted to singletons born after 37 weeks gestation as both multiple and premature birth is strongly associated with birth size and such infants are more prone to asphyxia and are at increased risk of later psychological morbidity. Gender-adjusted differences in the growth measures for those that did and did not attend the psychosis-like symptoms interview were examined with linear regression models. Pearson correlation coefficient was used to assess the linear relationship between birth weight and birth length. Logistic regression models were used to investigate the association of birth measures with both any suspected or definite psychosis-like symptoms item and definite psychosis-like symptoms positive cases only, after adjusting for age at the psychosis-like symptoms interview, gender and gestation; however, gender was not included in models which contained the gender-specific $z$-scores. Results are presented by odds ratio (OR) and 95\% confidence interval (95\% CI). Associations were also examined for narrowly defined psychosis-like symptoms and frequently occurring definite psychosis-like symptoms without attributions. The analysis was then repeated for the measures from the 7-year clinic, but gestation was not included in these models. Likelihood ratio tests (LRTs) were used to assess whether there was any evidence of non-linear effects, by including the quadratic terms for the z-scores of birth weight and ponderal index in the models. Each potential confounder was considered separately in the models to take account of the impact of changing sample sizes because of missing data for the different confounders assessed.

\section{Results}

\section{Study members}

Altogether 6455 children attended the psychosis-like symptoms interview. We excluded 172 children from multiple births and a further 283 children who were born before 37 weeks gestation. This resulted in a sample size of 6000 participants available for analysis (online Table DS1). There were 372 boys (12.7\%) and 448 girls 
$(14.6 \%)$ rated as having suspected or definite psychosis-like symptoms. The prevalence of definite psychosis-like symptoms was $144(4.9 \%)$ boys and 198 (6.5\%) girls. The mean birth weights were $3551 \mathrm{~g}$ and $3428 \mathrm{~g}$ for boys and girls respectively.

We have previously shown that cohort members who did not complete the psychosis-like symptoms interview were more likely to be male (54\% v. 49\%), have parents in a manual occupation $(55 \%$ v. $43 \%)$ and have less educated mothers (39\% v. $21 \%$ did not obtain any O-levels compared with those who were interviewed). ${ }^{5}$ Furthermore, cohort members who attended the psychosis-like symptoms interview had a (gender-adjusted) birth weight $43.6 \mathrm{~g}$ heavier than those that did not attend, and were $0.15 \mathrm{~cm}$ longer at birth. There were slight differences in the 7-year height and BMIs; those that attended the clinics were $0.33 \mathrm{~cm}$ shorter and had BMIs that were $0.28 \mathrm{~kg} / \mathrm{m}^{2}$ lower.

\section{Birth measures}

Associations of the three measures of fetal growth - birth weight, birth length and ponderal index - with psychosis-like symptoms are shown in online Table DS2. As there was no evidence that associations differed in males and females ( $P$ (interaction) ranged from $P=0.2$ to $P=0.9$ ), the data for males and females were combined. The models were initially adjusted for age at the psychosis-like symptoms interview and gender (where appropriate), and then gestation was also included in the models.

There were weak inverse associations of birth weight, birth length and ponderal index with any suspected or definite psychosis-like symptoms: the strongest association was with ponderal index.

Associations were stronger in relation to the definite psychosislike symptoms outcome. As birth weight, birth length and ponderal index increase, the odds of psychosis-like symptoms decline. There was an $18 \%$ reduction in the odds of psychosis-like symptoms in relation to a one standard deviation increase in birth weight in models controlling for age and gestation $(\mathrm{OR}=0.82$, 95\% CI 0.73-0.92, $P=0.001)$. The OR per $\mathrm{kg}$ increase in birth weight (results not presented in online Table DS2) was 0.65 (95\% CI 0.51-0.84). There was no statistical evidence of a non-linear association with birth weight (LRT $\chi^{2}=1.45, P=0.23$ ). The association of birth length with definite psychosis-like symptoms was similarly strong (OR per standard deviation increase $=0.84$, $95 \%$ CI $0.73-0.98, P=0.022)$. The OR per $10 \mathrm{~cm}$ increase in birth length (results not presented in online Table DS2) was 0.43 (95\% CI $0.20-0.89$ ). Associations were similar in relation to ponderal index (OR per standard deviation increase $=0.83$, 95\% CI $0.72-$ $0.96, P=0.011)$. There was weak statistical evidence of a non-linear association with ponderal index (LRT $\chi^{2}=3.08, P=0.08$ ), but no evidence of an increased risk in participants with a high ponderal index at birth. Associations were not changed when adjusting for resuscitation at birth, as a measure of birth asphyxia.

There was a strong correlation between birth weight and birth length $(r=0.79, P<0.001)$. We repeated the model for birth weight, additionally controlling for birth length and vice versa; the association of birth weight with psychosis-like symptoms was strengthened in the adjusted model (OR per standard deviation increase in birth weight $=0.73,95 \%$ CI $0.58-0.92$, $P=0.007)$ but the association with birth length was greatly attenuated $(\mathrm{OR}=1.07,95 \% \mathrm{CI} 0.85-1.34, P=0.57)$.

\section{Associations with narrowly defined symptoms and frequently occurring definite psychosis-like symptoms without attributions}

Associations of third person auditory hallucinations, first-rank delusions and narrowly defined psychosis-like symptoms with birth measures were in the same direction, but slightly weaker than those with the definite psychosis-like symptoms outcome. A one standard deviation increase in birth weight was associated with $11 \%$ (OR per standard deviation increase in birth weight $=0.89,95 \%$ CI $0.69-1.16, P=0.39), 13 \% \quad(\mathrm{OR}=0.87$, 95\% CI $0.73-1.03, P=0.10)$ and $12 \%(\mathrm{OR}=0.88,95 \%$ CI $0.76-$ $1.02, P=0.10)$ lower odds of each outcome. However, the lower prevalence $(1.1 \%, 2.9 \%$ and $3.7 \%$ respectively) of these end-points resulting from the more stringent classification meant that statistical power to detect differences was reduced. The association with frequently occurring definite psychosis-like symptoms without attributions was similar to the other psychosis-like symptoms outcomes for birth weight (OR per standard deviation increase $=0.77,95 \%$ CI $0.64-0.91, P=0.003$ ). The association for birth length was stronger than for previous psychosis-like symptoms outcomes (OR per standard deviation increase $=0.74$, 95\% CI $0.60-0.91, P=0.004)$.

\section{Adjusting for potential confounders}

Table 1 summarises the multivariable logistic regression models investigating the impact of a series of potential confounding factors on the association of the three birth measures with definite psychosis-like symptoms. Column A presents the fully adjusted ORs of each birth measure in relation to psychosis-like symptoms for the full data-set (already presented in online Table DS2). For each of the potential confounders/factors possibly on the causal pathway (IQ), the table initially presents the age- and gestationadjusted associations with psychosis-like symptoms in the subset of participants with complete data on the confounding factor of interest and psychosis-like symptoms (column B). Column C then shows the association for the birth measure with psychosis-like symptoms after controlling for each named confounder. Controlling for paternal height and weight, family history of schizophrenia, maternal and paternal age, or parity and household crowding did not change the effect estimates (results not presented).

The confounders that have an effect on the estimates are: maternal anthropometry, maternal smoking during pregnancy, parental socioeconomic status and IQ at age 8 years. Of the two measures of maternal anthropometry examined (maternal height and weight), it was adjustment for maternal height that was responsible for the attenuation. When the confounding effect of all these variables (maternal anthropometry, maternal smoking during pregnancy, socioeconomic status and IQ) in relation to the birth weight with psychosis-like symptoms association was assessed ( $n=4038$ participants), the OR per standard deviation change in birth weight attenuated from 0.90 (95\% CI 0.77-1.04) to 0.95 (95\% CI $0.81-1.11)$.

\section{Measures at age 7.5 years}

There was generally no statistical evidence for an association of height and adiposity measured at age 7.5 years with psychosis-like symptoms at age 12 years (Table 2). There was weak evidence that association of height with psychosis-like symptoms differed in males and females $(P$ (interaction $)=0.15)$. The OR per standard deviation increase in height was 0.87 (95\% CI $0.73-1.05$, $P=0.15)$ in males and 1.03 (95\% CI $0.88-1.20, P=0.74)$ in females. There was no statistical evidence for an association of height and adiposity for further psychosis-like symptoms outcomes of narrowly defined psychosis-like symptoms and frequently occurring definite psychosis-like symptoms without attributions. The effect estimates for size at 7.5 years did not change when adjusting for potential confounders (results not presented). 
Table 1 The association of indicators of fetal growth with psychosis-like symptoms (definite cases only) adjusting for potential confounders and factors possibly lying on causal pathways

\begin{tabular}{|c|c|c|c|c|c|c|c|c|c|}
\hline \multirow[b]{3}{*}{ z-scores } & \multirow[b]{3}{*}{ A } & \multicolumn{8}{|c|}{ Investigating the effects of controlling for specific confounders } \\
\hline & & \multicolumn{2}{|c|}{$\begin{array}{l}\text { Maternal height } \\
\text { and weight }\end{array}$} & \multicolumn{2}{|c|}{$\begin{array}{l}\text { Maternal smoking } \\
\text { during pregnancy }\end{array}$} & \multicolumn{2}{|c|}{$\begin{array}{c}\text { Markers of } \\
\text { socioeconomic status }\end{array}$} & \multicolumn{2}{|c|}{ IQ at 8 years } \\
\hline & & B & c & B & C & B & c & B & C \\
\hline \multicolumn{10}{|l|}{ Birth weight } \\
\hline$n$ & 5916 & 5363 & 5363 & 5865 & 5865 & 5348 & 5348 & 4694 & 4694 \\
\hline OR (95\% Cl) & 0.82 & 0.83 & 0.84 & 0.83 & 0.86 & 0.83 & 0.85 & 0.87 & 0.89 \\
\hline & $(0.73-0.92)$ & $(0.73-0.95)$ & $(0.74-0.96)$ & $(0.73-0.93)$ & $(0.76-0.97)$ & $(0.73-0.94)$ & $(0.74-0.96)$ & $(0.76-1.00)$ & $(0.78-1.02)$ \\
\hline$P$ & 0.001 & 0.006 & 0.012 & 0.002 & 0.015 & 0.005 & 0.011 & 0.051 & 0.095 \\
\hline \multicolumn{10}{|l|}{ Birth length } \\
\hline$n$ & 3929 & 3598 & 3598 & 3898 & 3898 & 3537 & 3537 & 3157 & 3157 \\
\hline OR $(95 \% \mathrm{Cl})$ & 0.84 & 0.84 & 0.88 & 0.85 & 0.89 & 0.84 & 0.87 & 0.85 & 0.87 \\
\hline & $(0.73-0.97)$ & $(0.73-0.99)$ & $(0.75-1.03)$ & $(0.73-0.98)$ & $(0.76-1.03)$ & $(0.72-0.99)$ & $(0.74-1.02)$ & $(0.72-1.01)$ & $(0.74-1.03)$ \\
\hline P & 0.022 & 0.033 & 0.125 & 0.028 & 0.126 & 0.033 & 0.078 & 0.063 & 0.107 \\
\hline \multicolumn{10}{|l|}{ Ponderal index } \\
\hline$n$ & 3882 & 3557 & 3557 & 3851 & 3851 & 3493 & 3493 & 3118 & 3118 \\
\hline OR $(95 \% \mathrm{Cl})$ & 0.83 & 0.81 & 0.80 & 0.82 & 0.83 & 0.81 & 0.80 & 0.87 & 0.86 \\
\hline & $(0.72-0.96)$ & $(0.69-0.94)$ & $(0.68-0.93)$ & $(0.71-0.95)$ & $(0.72-0.96)$ & $(0.69-0.95)$ & $(0.68-0.94)$ & $(0.73-1.02)$ & $(0.73-1.02)$ \\
\hline P & 0.011 & 0.006 & 0.005 & 0.009 & 0.012 & 0.009 & 0.050 & 0.089 & 0.085 \\
\hline
\end{tabular}

We looked at birth measures in relation to size at 7.5 years. There was no statistical evidence that associations for BMI differed in relation to birth weight $(P$ (interaction $)=0.87)$. For height at 7.5 years there was statistical evidence of an interaction with birth weight $(P$ (interaction $)=0.006)$. However, there was no clear patterning to stratified associations so this may be a chance finding. Statistical evidence that associations with birth length differed depending on height at age 7.5 was weak $(P($ interaction $)=0.14)$.

\section{Discussion}

\section{Summary of findings}

This analysis provides reasonably strong evidence that restricted fetal growth, indexed by low birth weight, short birth length and low ponderal index, are associated with an increased risk of interviewer-assessed psychosis-like symptoms at 12 years of age

\section{Table 2 The association (odds ratio, 95\% Cl) of measures of height and adiposity at age 7.5 years with psychosis-like symptoms ${ }^{a}$}

\begin{tabular}{|c|c|c|}
\hline & Any suspected or definite psychosis-like symptoms & Any definite psychosis-like symptoms \\
\hline \multicolumn{3}{|l|}{ 7.5-year height quintiles } \\
\hline 1 (Short) & $1.03(0.80-1.32)$ & $1.05(0.72-1.53)$ \\
\hline 2 & $0.99(0.77-1.27)$ & $1.11(0.77-1.62)$ \\
\hline 3 & $1.07(0.83-1.38)$ & $1.17(0.80-1.70)$ \\
\hline 4 & $1.00(0.78-1.28)$ & $0.90(0.61-1.33)$ \\
\hline 5 (Tall) & 1.00 & 1.00 \\
\hline$P$ & 0.97 & 0.69 \\
\hline 7.5-year height z-score & $0.99(0.91-1.07)$ & $0.96(0.85-1.08)$ \\
\hline$P$ & 0.75 & 0.52 \\
\hline \multicolumn{3}{|l|}{ 7.5-year waist circumference quintiles } \\
\hline 1 (Small) & $0.83(0.65-1.07)$ & $1.04(0.73-1.48)$ \\
\hline 2 & $0.78(0.60-1.00)$ & $0.78(0.53-1.15)$ \\
\hline 3 & $0.88(0.69-1.13)$ & $0.91(0.63-1.32)$ \\
\hline 4 & $0.83(0.64-1.06)$ & $0.76(0.52-1.12)$ \\
\hline 5 (Large) & 1.00 & 1.00 \\
\hline$P$ & 0.34 & 0.38 \\
\hline 7.5-year waist circumference $z$-score & $1.05(0.97-1.13)$ & $0.99(0.88-1.11)$ \\
\hline$P$ & 0.23 & 0.82 \\
\hline \multicolumn{3}{|l|}{ 7.5-year body mass index quintiles } \\
\hline 1 (LOW) & $0.95(0.74-1.22)$ & $1.05(0.71-1.54)$ \\
\hline 2 & $0.88(0.68-1.13)$ & $1.28(0.88-1.86)$ \\
\hline 3 & $0.87(0.68-1.12)$ & $1.03(0.69-1.52)$ \\
\hline 4 & $0.99(0.78-1.27)$ & $1.11(0.76-1.63)$ \\
\hline 5 (High) & 1.00 & 1.00 \\
\hline$P$ & 0.73 & 0.69 \\
\hline 7.5-year body mass index z-score & $1.04(0.96-1.12)$ & $0.98(0.87-1.11)$ \\
\hline$P$ & 0.37 & 0.75 \\
\hline
\end{tabular}


in the ALSPAC cohort. The study found no evidence of a relationship for postnatal body size and psychosis-like symptoms. All the associations for fetal growth were stronger in models investigating associations with definite psychosis-like symptoms compared with those using the combined category of suspected or definite psychosis-like symptoms. These associations were somewhat attenuated in models controlling for maternal size, socioeconomic status and the child's IQ. Since adjusting for IQ only slightly attenuates the association of birth weight with psychosis-like symptoms, cognitive function may not lie on the causal pathway linking these two factors. The association with birth length was confounded by birth weight; no independent association with birth length was seen in models controlling for birth weight. There is high correlation between the birth length and birth weight $(r=0.79)$. It is likely that birth length is measured with more error than birth weight and so the 'independent' effect of birth weight may simply be because it is measured with less error. ${ }^{20}$ We found no evidence of an association between childhood height, BMI or waist circumference and psychosis-like symptoms.

\section{Strengths and limitations}

There are several strengths to our study. First, ALSPAC is a large population-based cohort and because of the large sample size, we had sufficient power to detect potentially important associations. Second, as fetal and childhood growth were recorded prospectively and were based on clinical measurements (rather than being self-reported), recall bias is not an issue and the possibility of measurement error is reduced. Lastly, careful quality control procedures were adopted to ensure psychosis-like symptoms were reliably recorded: in associated reliability studies the average overall interrater reliability was very good $(\kappa=0.72){ }^{5}$

The main limitation to this study is the current uncertainty concerning the clinical significance of self-reported psychosis-like symptoms in childhood and its association with clinical psychosis. The Dunedin cohort is the only study to have examined this association to date. ${ }^{1}$ Further follow-up of ALSPAC and other cohorts will elucidate this issue. Another drawback of our study is attrition from the cohort over time, with levels of losses to follow-up similar to those seen in other large-scale longitudinal studies. ${ }^{21,22}$ As heavier babies were more likely to attend the clinic where psychosis-like symptoms were measured, this could result in an underestimate of the birth weight and birth length associations observed, if children experiencing psychosis-like symptoms are less likely to attend the clinics. An additional limitation to this study is that although information was available on family history of schizophrenia, there was no information regarding family histories of other Axis I disorders. Another limitation was that because of organisational constraints on the interview time we focused entirely on the careful measurement of psychosis-like symptoms. It was therefore not possible to extend the interview with other measures such as interpersonal functioning and clinical treatment history, and it was not thus possible to identify those psychosis-like symptom-positive children who may have had a fully developed childhood schizophrenic or other psychotic clinical disorder. However, given the extremely low prevalence of childhood psychosis ${ }^{23}$ we do not think this influences the validity of our findings.

\section{Comparison with other studies}

Associations of low birth weight $(<2.0 \mathrm{~kg} /<2.5 \mathrm{~kg})$ with an increased risk of schizophrenia have been reported in a number of studies (see review by Cannon et al). ${ }^{9}$ However, the largest study of the relationship between birth weight and clinically treated schizophrenia, based on 736 incident cases identified within a Swedish record-linkage study of psychiatric hospital admissions, found no evidence of such an association. ${ }^{7}$ Birth length was, however, inversely related to risk in the same study; the OR per $10 \mathrm{~cm}$ increase in birth length was 0.53 (95\% CI $0.31-0.89),{ }^{7}$ similar to that seen in the present study in relation to psychosis-like symptoms ( $\mathrm{OR}=0.43,95 \%$ CI $0.20-0.89)$. It should be noted that a $10 \mathrm{~cm}$ increase in birth length is a large change (approximately 5 standard deviations) so the magnitude (and clinical relevance) of this association should be interpreted cautiously. In the Swedish study, in contrast to our analysis of ALSPAC participants, birth length-schizophrenia associations were not attenuated when birth weight was controlled for in the multivariable models. In a previous analysis of ALSPAC data, Wiles et al found that short birth length, but not birth weight, was associated with an increased risk of behavioural difficulties at age 7 years. ${ }^{24}$

An emerging body of literature suggests that low BMI in child$\operatorname{hood}^{8}$ and early adulthood ${ }^{7,25,26}$ is associated with an increased risk of psychosis. Furthermore, short height in adulthood ${ }^{7}$ but not childhood ${ }^{8}$ has also been shown to be associated with an increased risk of psychosis. However, we found no real evidence of an association with BMI or height in our analysis. If psychosis-like symptoms are indeed a marker of later risk of psychosis, the mismatch between the associations of our childhood measures of height and BMI with psychosis-like symptoms compared with those seen in the literature could be as a result of our limited power to detect such relatively weak associations, or because factors influencing height and weight are important in determining which people with psychosis-like symptoms go on to develop psychosis, rather than being of importance in the aetiology of psychosis-like symptoms per se.

\section{Aetiology of psychosis-like symptoms and implications for treatment and prognosis}

A growing body of scientific evidence indicates that schizophrenia is a disorder of neurodevelopment and brain maturation. ${ }^{27}$ Psychosis-like symptoms may be an important component of a pan-developmental process and eventually, as these children are followed up further, could be associated with 'prodomal' indicators such as decline and withdrawal. Our findings of an association between measures of fetal growth and psychosis-like symptoms provide some support for their status as developmental antecedents of adult-onset psychosis, with a possible common origin in prenatal development factors. However, notwithstanding the association with fetal growth reported here, it has been suggested that psychosis-like symptoms may be a general indicator of emotional problems in childhood that act as a risk factor for later psychosis, rather than being part of a developmental 'continuum' with adult-onset schizophrenia (details available from the author on request). These findings at age 12 years are especially intriguing because the participants have not passed through adolescence and all of the neuromaturational factors potentially contributing to an exacerbation in psychosis-like symptoms (e.g. synaptic pruning, heightened cortisol) have not occurred. The long-term clinical consequences of experiencing psychosis-like symptoms in childhood remain uncertain. In the Dunedin cohort the presence of psychosis-like symptoms at age 11 was associated with a fivefold risk of later psychosis. Further studies of this issue are required and long-term follow-up of the ALSPAC cohort - a study approximately ten times larger than Dunedin - will help clarify the aetiological, and hence clinical, significance of psychosis-like symptoms. Our study adds to the growing body of literature examining psychosis-like symptoms in the ALSPAC cohort. $^{5,28,29}$ 
These findings provide mixed evidence to support commonality in associations of birth and childhood anthropometry with psychosis-like symptoms at 12 years of age and adult-onset psychosis. The results indicate support for associations of fetal growth and psychosis-like symptoms, but not for postnatal exposures and psychosis-like symptoms at 12 years. As birth weight associations were only partially attenuated in models controlling for IQ, it seems likely that cognitive function does not lie on the causal pathway between prenatal influences on growth and psychosis-like symptoms.

K. Thomas, MSC, Department of Social Medicine, University of Bristol;

G. Harrison, MD, FRCPsych, Academic Unit of Psychiatry, University of Bristol;

S. Zammit, PhD, Academic Unit of Psychiatry, University of Bristol, and Department

of Psychological Medicine, Cardiff University; G. Lewis, FRCPsych, PhD, Academic

Unit of Psychiatry, University of Bristol; J. Horwood, BSC, J. Heron, PhD, Department

of Social Medicine, University of Bristol; C. Hollis, PhD, DCH, MRCPsych, Division of

Psychiatry, University of Nottingham; D. Wolke, Dipl Psych, PhD, Department of

Psychology, University of Warwick; A. Thompson, MD, MRCPsych, Academic Unit of

Psychiatry, University of Bristol; D. Gunnell, PhD, FFPH, Department of Social

Medicine, University of Bristol, UK.

Correspondence: David Gunnell, Department of Social Medicine, University

of Bristol, Canynge Hall, 39 Whatley Road, Bristol BS8 2PS, UK. Email:

D.J.Gunnell@bristol.ac.uk

First received 25 Feb 2008, final revision 22 Sep 2008, accepted 3 Dec 2008

\section{Funding}

The UK Medical Research Council, the Wellcome Trust and the University of Bristol provide core support for ALSPAC. This research was funded by the Wellcome Trust; grant no. 072043

\section{Acknowledgements}

We are extremely grateful to all the families who took part in this study, the midwives for their help in recruiting them, and the entire ALSPAC team. All the authors had full access to all of the data in the study and take responsibility for the integrity of the data and the accuracy of the data analysis. This publication is the work of the authors, who will serve as guarantors for the contents of this paper.

\section{References}

1 Poulton R, Caspi A, Moffitt TE, Cannon M, Murray R, Harrington H. Children's self-reported psychotic symptoms and adult schizophreniform disorder: a 15-year longitudinal study. Arch Gen Psychiatry 2000; 57: 1053-8.

2 Johns LC, Cannon M, Singleton N, Murray RM, Farrell M, Brugha T, et al. Prevalence and correlates of self-reported psychotic symptoms in the British population. Br J Psychiatry 2004; 185: 298-305.

3 Van Os J, Hanssen M, Bijl RV, Vollebergh W. Prevalence of psychotic disorde and community level of psychotic symptoms: an urban-rural comparison. Arch Gen Psychiatry 2001; 58: 663-8.

4 Hanssen $\mathrm{M}$, Bak $\mathrm{M}$, Bijl R, Vollebergh $\mathrm{W}$, van OJ. The incidence and outcome of subclinical psychotic experiences in the general population. $\mathrm{Br} J \mathrm{Clin}$ Psychol 2005; 44: 181-91.

5 Horwood J, Salvi G, Thomas K, Duffy L, Gunnell D, Hollis C, et al. IQ and nonclinical psychotic symptoms in 12-year-olds: results from the ALSPAC birth cohort. Br J Psychiatry 2008; 193: 185-91.

6 Cannon M, Caspi A, Moffitt TE, Harrington H, Taylor A, Murray RM, et al. Evidence for early-childhood, pan-developmental impairment specific to schizophreniform disorder: results from a longitudinal birth cohort. Arch Gen Psychiatry 2002; 59: 449-56.

7 Gunnell D, Harrison G, Whitley E, Lewis G, Tynelius P, Rasmussen F. The association of fetal and childhood growth with risk of schizophrenia. Cohort study of 720,000 Swedish men and women. Schizophr Res 2005; 79: 315-22.
8 Wahlbeck K, Forsen T, Osmond C, Barker DJ, Eriksson JG. Association of schizophrenia with low maternal body mass index, small size at birth, and thinness during childhood. Arch Gen Psychiatry 2001; 58: 48-52.

9 Cannon $\mathrm{M}$, Jones PB, Murray RM. Obstetric complications and schizophrenia: an historical and meta-analytic review. Am J Psychiatry 2002; 159: 1080-92.

10 Weinberger DR. Implications of normal brain development for the pathogenesis of schizophrenia. Arch Gen Psychiatry 1987; 44: 660-9.

11 Golding J, Pembrey M, Jones R, ALSPAC study team. ALSPAC - the Avon Longitudinal Study of Parents and Children. I. Study methodology. Paediatr Perinat Epidemiology 2001; 15: 74-87.

12 American Psychiatric Association. Diagnostic and Statistical Manual of Mental Disorders (4th edn) (DSM-IV). APA, 1994.

13 World Health Organization. International Statistical Classification of Diseases and Related Health Problems, Tenth Revision (ICD-10) (2nd edn). WHO, 2005.

14 Cameron N. The methods of auxological anthropometry. In Human Growth. A Comprehensive Treatise (3rd edn) (ed F Falkner): 3-46. Plenum Press, 1986.

15 Office of Population Censuses and Surveys. Standard Occupational Classification. HMSO, 1991.

16 Wechsler D, Golombok S, Rust J. Wechsler Intelligence Scale for Children (3rd edn) (WISC-III-UK). Psychological Corporation, 1992.

17 Richards M, Hardy R, Kuh D, Wadsworth ME. Birth weight and cognitive function in the British 1946 birth cohort: Iongitudinal population based study. BMJ 2001; 322: 199-203.

18 David AS, Malmberg A, Brandt L, Allebeck P, Lewis G. IQ and risk for schizophrenia: a population-based cohort study. Psychol Med 1997; 27: 1311-23.

19 Gunnell D, Harrison G, Rasmussen F, Fouskakis D, Tynelius P. Associations between premorbid intellectual performance, early-life exposures and earlyonset schizophrenia. Cohort study. Br J Psychiatry 2003; 181: 298-305.

20 Phillips AN, Davey Smith G. How independent are 'independent' effects? Relative risk estimation when correlated exposures are measured imprecisely. J Clin Epidemiol 1991; 44: 1223-31.

21 Callaway LK, McIntyre HD, O'Callaghan M, Williams GM, Najman JM Lawlor DA. The association of hypertensive disorders of pregnancy with weight gain over the subsequent 21 years: findings from a prospective cohort study. Am J Epidemiol 2007; 166: 421-8.

22 Plewis I, Calderwoof L, Hawkes D, Nathan G. National Child Development Study and 1970 British Cohort Study Technical Report: Changes in the NCDS and BCS70 populations and samples over time. Centre for Longitudinal Studies, Institute of Education, 2004.

23 Gillberg C. Infantile autism and other childhood psychoses in a Swedish urban region. Epidemiological aspects. J Child Psychol Psychiatry 1984; 25 $35-43$.

24 Wiles NJ, Peters TJ, Heron J, Gunnell D, Emond A, Lewis G. Fetal growth and childhood behavioral problems: results from the ALSPAC cohort. Am J Epidemiol 2006; 163: 829-37.

25 Zammit S, Rasmussen F, Farahmand B, Gunnell D, Lewis G, Tynelius P, et al. Height and body mass index in young adulthood and risk of schizophrenia: $a$ longitudinal study of 1347520 Swedish men. Acta Psychiatr Scand 2007; 116: $378-85$.

26 Weiser M, Knobler $\mathrm{H}$, Lubin G, Nahon D, Kravitz E, Caspi A, et al. Body mass index and future schizophrenia in Israeli male adolescents. J Clin Psychiatry 2004; 65: 1546-9.

27 Harrison PJ. The neuropathology of schizophrenia. A critical review of the data and their interpretation. Brain 1999; 122: 593-624.

28 Zammit S, Horwood J, Thompson A, Thomas K, Menezes P, Gunnell D, et al. Investigating if psychosis-like symptoms (PLIKS) are associated with family history of schizophrenia or paternal age in the ALSPAC birth cohort. Schizophr Res 2008; 104: 279-86.

29 Zammit S, Odd D, Horwood J, Thompson A, Thomas K, Menezes P et al. Investigating whether adverse prenatal and perinatal events are associated with non-clinical psychotic symptoms at age 12 years in the ALSPAC birth cohort. Psychol Med 2009; 12 February: doi: 10.1017/ S0033291708005126. 